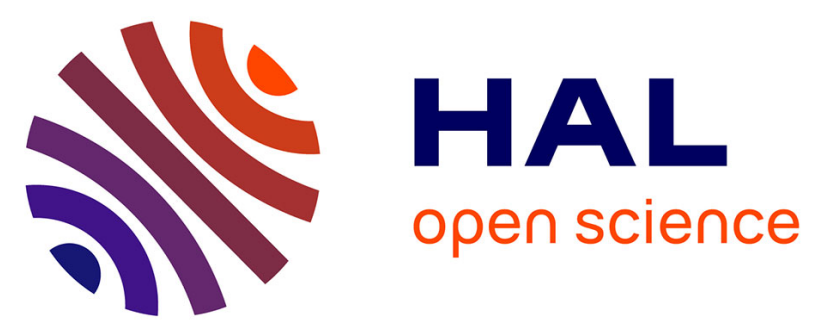

\title{
Investigation of phase-contrast magnetic resonance imaging underestimation of turbulent flow through the aortic valve phantom: Experimental and computational study using lattice Boltzmann method
}

Radek Fučík, Radek Galabov, Petr Pauš, Pavel Eichler, Jakub Klinkovský, Robert Straka, Jaroslav Tintěra, Radomir Chabiniok

\section{To cite this version:}

Radek Fučík, Radek Galabov, Petr Pauš, Pavel Eichler, Jakub Klinkovský, et al.. Investigation of phase-contrast magnetic resonance imaging underestimation of turbulent flow through the aortic valve phantom: Experimental and computational study using lattice Boltzmann method. Magnetic Resonance Materials in Physics, Biology and Medicine, 2020, 33 (5), pp.649-662. hal-02470588v2

\author{
HAL Id: hal-02470588 \\ https://hal.inria.fr/hal-02470588v2
}

Submitted on 4 Mar 2020

HAL is a multi-disciplinary open access archive for the deposit and dissemination of scientific research documents, whether they are published or not. The documents may come from teaching and research institutions in France or abroad, or from public or private research centers.
L'archive ouverte pluridisciplinaire HAL, est destinée au dépôt et à la diffusion de documents scientifiques de niveau recherche, publiés ou non, émanant des établissements d'enseignement et de recherche français ou étrangers, des laboratoires publics ou privés. 


\title{
Investigation of phase-contrast magnetic resonance imaging underestimation of turbulent flow through the aortic valve phantom: Experimental and computational study using lattice Boltzmann method
}

\author{
Radek Fučík - Radek Galabov - Petr Pauš • Pavel Eichler · Jakub \\ Klinkovský • Robert Straka • Jaroslav Tintěra • Radomír Chabiniok
}

Received: date / Accepted: date

The work was supported by the Ministry of Health of the Czech Republic project No. NV19-08-00071, Czech Science Foundation project No. 18-09539S, OPVVV project No. CZ.02.1.01/0.0/0.0/16_019/0000765, the Wellcome/EPSRC Centre for Medical Engineering (WT 203148/Z/16/Z), and by the Inria-UT Southwestern Associated Team TOFMOD. In addition, the authors would like to thank IBM Watson iLab for providing access to a system based on IBM Power9 CPUs.

\section{R. Fučík}

Faculty of Nuclear Sciences and Physical Engineering, Czech Technical University in Prague, Trojanova 13, 12000 Praha 2, Czech Republic

Tel.: +420224358540

E-mail: fucik@fjfi.cvut.cz

\section{R. Galabov}

Faculty of Nuclear Sciences and Physical Engineering, Czech Technical University in Prague, Trojanova 13, 12000 Praha 2, Czech Republic

Institute of Clinical and Experimental Medicine, Prague, Czech Republic

P. Pauš

Faculty of Nuclear Sciences and Physical Engineering, Czech Technical University in Prague, Trojanova 13, 12000 Praha

2, Czech Republic

P. Eichler

Faculty of Nuclear Sciences and Physical Engineering, Czech Technical University in Prague, Trojanova 13, 12000 Praha

2, Czech Republic

J. Klinkovský

Faculty of Nuclear Sciences and Physical Engineering, Czech Technical University in Prague, Trojanova 13, 12000 Praha

2, Czech Republic

R. Straka

Department of Heat Engineering and Environment Protection, AGH University of Science and Technology in Kraków, Poland

Faculty of Nuclear Sciences and Physical Engineering, Czech Technical University in Prague, Trojanova 13, 12000 Praha

2, Czech Republic

\begin{abstract}
Objective The accuracy of phase-contrast magnetic resonance imaging (PC-MRI) measurement is investigated using a computational fluid dynamics (CFD) model with the objective to determine the magnitude of the flow underestimation due to turbulence behind a narrowed valve in a phantom experiment.
\end{abstract}

Materials and Methods An acrylic stationary flow phantom is used with three insertable plates mimicking aortic valvular stenoses of varying degrees. Positive and negative horizontal fluxes are measured at equidistant slices using standard PC-MRI sequences by $1.5 \mathrm{~T}$ and $3 \mathrm{~T}$ systems. The CFD model is based on the 3D lattice Boltzmann method (LBM). The experimental and simulated data are compared using the Bland-Altmanderived limits of agreement. Based on the LBM results, the turbulence is quantified and confronted with the level of flow underestimation.

Results LBM gives comparable results to PC-MRI for valves up to moderate stenosis on both field strengths. The flow magnitude through a severely stenotic valve was underestimated due to signal void in the regions of turbulent flow behind the valve, consistently with the level of quantified turbulence intensity.

\section{J. Tintěra}

Institute of Clinical and Experimental Medicine, Prague, Czech Republic

\section{R. Chabiniok}

Inria, France

LMS, Ecole Polytechnique, CNRS, Institut Polytechnique de Paris, France

School of Biomedical Engineering \& Imaging Sciences (BMEIS), St. Thomas Hospital, King's College London, UK Faculty of Nuclear Sciences and Physical Engineering, Czech Technical University in Prague, Trojanova 13, 12000 Praha 2, Czech Republic

Division of Pediatric Cardiology, UT Southwestern Medical Center Dallas, TX 75235-7701, USA 
Discussion Flow measured by PC-MRI is affected by noise and turbulence. LBM can simulate turbulent flow efficiently and accurately, it has therefore the potential to improve clinical interpretation of PC-MRI.

Keywords phase-contrast MRI - flow phantom . computational fluid dynamics · lattice Boltzmann method $\cdot$ aortic valve stenosis

\section{Introduction}

The quantitative assessment of valvular diseases - assessing the level of stenosis or regurgitation - represents the most frequent application of phase-contrast magnetic resonance imaging (PC-MRI) [29]. Even though echocardiography is usually the method of choice, there exist situations where the results are inconclusive or the patients' hearts are not sufficiently accessible by ultrasonography. In these cases the PC-MRI contributes to the diagnosis [11]. The underestimation of turbulent flow by PC-MRI is a known issue and a limited quantification of the measurement error is a major drawback [30]. Furthermore, in the quantification of valvular regurgitation, there is an unresolved question regarding the position of the slice for measurements of the forward and backward flow since the presence of turbulence and the motion of valve complicate its positioning [29]. Different authors choose different sites for their measurements $[36,23,7,4,6,26]$. Using the 4D PC-MRI in conjunction with subsequent post-processing might produce better results [11]. However, acquiring 4D flow sequences substantially prolongs the MRI exam. This paper therefore focuses on the widely accessible and routinely used 2D PC-MRI.

Aortic valve stenosis is usually assessed by the peak velocity [29, 11]. A key parameter used for stenosis assessment is the pressure drop computed from the velocity in front of and behind the valve. Under the assumption of laminar flow, a simple Bernoulli model is typically employed. Since the pressure drop depends not only on the kinetic energy loss but also on the losses due to turbulence, the obtained value underestimates the real pressure difference $[22,38,10]$. Extensions of the method were proposed to address the underestimation $[39,40]$. Another method proposed in [20] estimates the real pressure drop based on 4D flow MRI coupled with a computational fluid dynamics (CFD) model, demonstrating its potential use in clinical practice.

The use of CFD in cardiovascular imaging has been recently investigated in several studies, especially coupled with PC-MRI [37]. Possible CFD applications in cardiovascular medicine include, for example, the diagnosis and treatment of aortic or cerebral aneurysm
[1], aortic coarctation [20], vessel or valvular stenosis $[22,25]$, valvular regurgitation [41], or a description of the aortic arch flow [27]. The flow data obtained by the PC-MRI can serve as a boundary condition for the CFD model [29] and the anatomy extracted from a cardiovascular MRI examination provides a geometrical boundary condition. The validated CFD model is then expected to detect, localize, and quantitatively describe a suspected lesion by investigating suitable hemodynamic parameters such as wall shear stress or energy loss [27]. The next step is the in silico simulation of potential interventional approaches or quantification of the risk, if the intervention is deferred. It can be foreseen that in the future, CFD will be implemented as a routine part of clinical examinations and assessment of treatment options suited for individual patients, although larger studies proving the reliability of the method are yet to be performed [28].

The scope of this article is to use a CFD model to investigate and quantify the in vitro underestimation of forward and backward flows measured by PC-MRI due to the presence of turbulence behind a stenosed valve or a narrowed vessel region. The CFD model used is based on the 3D lattice Boltzmann method (LBM, [21]) in its state-of-the-art variant $[21,9,17,18,19,16,8,24]$, which was shown to be suitable for both laminar and turbulent Newtonian blood flows in large arteries $[14,3]$ and efficient to simulate transitions from laminar to turbulent flow without any specific turbulence model, i.e. without the need of adjusting any additional parameters specific to the turbulent regime [15].

To achieve these objectives, a specifically designed MRI flow-phantom experiment was carried out, while the LBM CFD model [14] developed previously in our lab was employed. The flow-phantom allowed the variation of inflow and interchangeable valve segments were used to represent the aortic valve with stenosis of various degrees and morphological types.

\section{Materials and methods}

\subsection{Experimental method}

The MRI experiments were performed on an acrylic flow phantom employed in a circulatory system, as shown in Figure 1. The rigid phantom was connected to a clean water reservoir using rubber hoses. Water was supplied into the system by an aquarium pump (Compact plus 5000, EHEIM, Deizisau, Germany) under a constant flow rate. A mechanical flow meter (FCH-midi-POM 97478940, BIO-TECH, Vilshofen, Germany) and a clamp enabling flow rate regulation were included. Three insertable rigid plates shown in Figure 2 represented aor- 
tic valve with stenosis of various degrees and morphology and induced various types of flow in the phantom.

The phantom is made from a rigid acrylic tube surrounded by a copper sulfate solution to provide a strong signal for a subsequent background phase correction. To allow the insertion of the valve plate, the tube have three separate parts. Additionally, another acrylic tube is inserted prior to the phantom inlet to ensure stabilized flow entering the phantom. The four parts of the tube are 1500.0/145.8/47.1/145.8 $\mathrm{mm}$ in length, see Figure 1. The inner diameters are 26.0/26.5/26.0/26.5 $\mathrm{mm}$. The circular valve plate of $3.9 \mathrm{~mm}$ in thickness is inserted between the second and the third part of the tube downstream. Rings of rubber hose are used to coat and seal the transitions between the parts.

The rigid acrylic plates with a laser-cut orifice shown in Figure 2 mimic mild (Mild-VS), moderate (Mod-VS), and severe (Sev-VS) valvular stenoses. Four flow rates of $65,91,129$, and $303 \mathrm{ml} / \mathrm{s}$ were used. While the first three represent the cardiac output values at rest, the highest flow rate allows investigating the regime of an increased cardiac output.

The PC-MRI experiments were conducted on two MRI scanners by the same vendor (Siemens Healthineers, Erlangen, Germany): Avanto Fit 1.5T and Trio $3 \mathrm{~T}$ MRI scanners. Both scanners have a maximum gradient strength of $45 \mathrm{mT} / \mathrm{m}$ and a maximum slew rate of $200 \mathrm{~T} / \mathrm{m} / \mathrm{s}$. Body array coils with 18 and 6 channels were used with the $1.5 \mathrm{~T}$ and $3 \mathrm{~T}$ scanner, respectively, while the spine matrix coil was also activated in both cases. Equidistantly spaced 2D slices, perpendicular to the phantom tube, were acquired by a phase-contrast sequence with Cartesian readout. The tube was parallel to the long axis of the scanner, and the valve plate was placed as close to the isocenter as possible. In the case of the $1.5 \mathrm{~T}$ and $3 \mathrm{~T}$ measurements, the stack of 25 and 26 adjacent slices covered a 10.0- and 10.4-cmlong region along the tube, respectively. The sequence parameters were set to $\mathrm{TE} / \mathrm{TR}=2.9 / 51.3 \mathrm{~ms}$, flip angle $15^{\circ}$, and slice thickness of $4 \mathrm{~mm}$. The field-of-view (FOV), spatial resolution, pixel size and velocity encoding range (VENC) are listed in Table 1. To ensure a high degree of similarity to clinical imaging, despite the constant flow rate, a retrospective triggering was used with a "dummy" heartbeat interval of $1000 \mathrm{~ms}$ and 40 calculated phases (20 actually measured phases) per heartbeat, i.e., the reconstructed and measured temporal resolutions were 25 and $50 \mathrm{~ms}$, respectively. The VENC parameter was adjusted to avoid velocity aliasing and to preserve the optimal velocity-to-noise ratio. To create a large enough statistical sample, each measurement was repeated 10 and 5 times for the $1.5 \mathrm{~T}$ and $3 \mathrm{~T}$ systems, respectively.
All acquired data was post-processed using Matlab (MathWorks, Natick, MA, USA). Since the flow was not pulsatile, 400 and 200 images corresponding to 10 and 5 repeated acquisitions of 40 phases of the dummy heart cycle for $1.5 \mathrm{~T}$ and $3 \mathrm{~T}$ systems, respectively, were treated the same way. For each slice, a circular region of interest (ROI) of $27.0 \mathrm{~mm}$ in diameter was delineated on a fused magnitude-velocity image. The eddy current correction was performed by fitting a quadratic surface through the static background surrounding the tube and subtracting the interpolated data from the velocities in the ROI. The mean velocity for each pixel in each slice was obtained by averaging all velocity measurements at a given location. The flux was computed by integrating all velocities across the ROI.

\subsection{Mathematical model and computational method}

The test section of the aortic phantom, in which the flow characteristics are acquired by the PC-MRI, is represented by a $3 \mathrm{D}$ computational domain $\Omega=\left[0, L_{x}\right] \times$ $\left[0, L_{y}\right] \times\left[0, L_{z}\right]$, as shown in Figure 1 . The boundary of $\Omega$ is denoted by $\Gamma$ and consists of the inflow, outflow, and impermeable wall boundaries $\Gamma_{i n}, \Gamma_{\text {out }}$, and $\Gamma_{w a l l}$. At $\Gamma_{\text {wall }}$, the no-slip condition using the bounce-back boundary condition for LBM is prescribed [21].

The governing equations of the CFD model in $\Omega$ and a time interval $[0, T]$ are given by the Navier-Stokes equations for an incompressible Newtonian fluid. The computational domain $\Omega$ is discretized by using an equidistant lattice $\Omega_{h}$ with a grid size $h$ representing the distance between the adjacent lattice sites.

The numerical approximation of the fluid density $\rho$ and velocity $\mathbf{v}$ is made by using LBM [21]. LBM is based on the mesoscopic description of fluid dynamics using discrete particle probability density functions $f_{i}=f_{i}(\mathbf{x}, t), \mathbf{x} \in \Omega_{h}, i=1,2, \ldots, q$, where $q$ denotes the number of discrete directions, in which the information can be spread. In this study, the popular 3D model with $q=27$, commonly denoted by D3Q27 [21], is used. For all $i=1,2, \ldots, q$, functions $f_{i}$ satisfy the discrete lattice Boltzmann equation in the form

$f_{i}\left(\mathbf{x}+\Delta t \boldsymbol{\xi}_{i}, t+\Delta t\right)-f_{i}(\mathbf{x}, t)=C_{i}(\mathbf{x}, t)$,

where $\Delta t$ is the lattice time step, $\boldsymbol{\xi}_{i}$ denotes the discrete lattice velocity vector, $\mathbf{x} \in \Omega_{h}$, and $C_{i}$ is the discrete collision operator. From the discrete probability density functions, macroscopic quantities, such as the fluid density $\rho$ and velocity $\mathbf{v}$, can be recovered as $\rho=\sum_{i=1}^{q} f_{i}$ and $\mathbf{v}=\sum_{i=1}^{q} f_{i} \boldsymbol{\xi}_{i}$, respectively.

The LBM numerical scheme consists of a collision and a streaming step. In the collision step, the collision operator $C_{i}$ is evaluated based on the values of $f_{i}$ 


\begin{tabular}{|c|c|c|c|c|c|c|c|}
\hline \multirow{2}{*}{$\begin{array}{l}\text { Valve identification } \\
\text { Description }\end{array}$} & & \multicolumn{2}{|c|}{ Mild-VS } & \multicolumn{2}{|c|}{ Mod-VS } & \multicolumn{2}{|c|}{ Sev-VS } \\
\hline & & $\begin{array}{r}\text { mild } \mathrm{v} \\
\text { sten }\end{array}$ & $\begin{array}{l}\text { alvular } \\
\text { osis }\end{array}$ & $\begin{array}{r}\text { moderate } \\
\text { ster }\end{array}$ & $\begin{array}{l}\text { valvular } \\
\text { osis }\end{array}$ & $\begin{array}{r}\text { severe } \\
\text { ster }\end{array}$ & $\begin{array}{l}\text { alvular } \\
\text { osis }\end{array}$ \\
\hline \multicolumn{8}{|l|}{ Shape } \\
\hline Field strength $B_{0}$ & {$[\mathrm{~T}]$} & 1.5 & 3.0 & 1.5 & 3.0 & 1.5 & 3.0 \\
\hline Wetted perimeter $P_{0}$ & {$[\mathrm{~mm}]$} & 58.9 & 58.9 & 44.4 & 44.4 & 35.5 & 35.5 \\
\hline Pixel size (in plane) & {$[\mathrm{mm}]$} & 1.14 & 1.14 & 1.14 & 1.14 & 1.14 & 1.14 \\
\hline Slice thickness & {$[\mathrm{mm}]$} & 4 & 4 & 4 & 4 & 4 & 4 \\
\hline $\mathrm{FOV}$ & {$[\mathrm{mm}]$} & $95 \times 254$ & $82 \times 292$ & $95 \times 254$ & $82 \times 292$ & $95 \times 254$ & $82 \times 292$ \\
\hline Matrix & & $224 \times 84$ & $256 \times 72$ & $224 \times 84$ & $256 \times 72$ & $224 \times 84$ & $256 \times 72$ \\
\hline \multicolumn{8}{|l|}{ Flow rate $65 \mathrm{ml} / \mathrm{s}$ : } \\
\hline VENC & {$[\mathrm{cm} / \mathrm{s}]$} & 50 & 75 & 50 & 75 & 100 & 100 \\
\hline$v_{0}$ & {$[\mathrm{~cm} / \mathrm{s}]$} & 15.75 & 15.72 & 15.75 & 15.22 & 17.93 & 15.68 \\
\hline $\operatorname{Re}$ & & 4414 & 4414 & 5853 & 5853 & 7325 & 7325 \\
\hline \multicolumn{8}{|l|}{ Flow rate $91 \mathrm{ml} / \mathrm{s}$ : } \\
\hline VENC & {$[\mathrm{cm} / \mathrm{s}]$} & 75 & 75 & 75 & 75 & 175 & 175 \\
\hline$v_{0}$ & {$[\mathrm{~cm} / \mathrm{s}]$} & 22.38 & 21.69 & 22.02 & 21.00 & 25.15 & 21.46 \\
\hline $\operatorname{Re}$ & & 6180 & 6180 & 8194 & 8194 & 10254 & 10254 \\
\hline \multicolumn{8}{|l|}{ Flow rate $129 \mathrm{ml} / \mathrm{s}$ : } \\
\hline VENC & {$[\mathrm{cm} / \mathrm{s}]$} & 100 & 75 & 110 & 110 & 200 & 200 \\
\hline$v_{0}$ & {$[\mathrm{~cm} / \mathrm{s}]$} & 30.92 & 28.88 & 30.84 & 27.85 & 34.07 & 28.41 \\
\hline $\operatorname{Re}$ & & 8761 & 8761 & 11616 & 11616 & 14536 & 14536 \\
\hline \multicolumn{8}{|l|}{ Flow rate $91 \mathrm{ml} / \mathrm{s}$ : } \\
\hline VENC & {$[\mathrm{cm} / \mathrm{s}]$} & 350 & 200 & 400 & 375 & 600 & 600 \\
\hline$v_{0}$ & {$[\mathrm{~cm} / \mathrm{s}]$} & 73.70 & 73.94 & 75.31 & 73.39 & 83.53 & 71.43 \\
\hline $\operatorname{Re}$ & & 20577 & 20577 & 27285 & 27285 & 34144 & 34144 \\
\hline
\end{tabular}

Table 1 The parameters of PC-MRI sequence for the measurements on the three valves (including the velocity encoding range VENC), measured maximal inflow velocities $v_{0}$, and Reynolds numbers (Re) computed for all four flow rates. Note that $v_{0}$ represents maximal velocity of the stable laminar profile before the valve while VENC is set to be higher than the expected maximum velocity throughout the whole phantom.

from the previous time step. In the subsequent streaming step, the post-collision state is transferred to the neighboring grid nodes in the direction of $\boldsymbol{\xi}_{i}$ for all $i=1,2, \ldots, q$. Since the streaming step is linear and involves only a single computer memory read and write operation per discrete probability density function $f_{i}$, and the collision step involves only local operations, the computational algorithm can be easily parallelized and is suitable for computation on Graphics Processing Units (GPUs). This allows massive parallelization and efficient computation even on a common computer equipped with a suitable GPU.

Several variants for the collision operator $C_{i}$ have been developed, such as SRT-LBM (single relaxation time LBM) [21], MRT-LBM (multiple relaxation time
LBM) [9], CuLBM (Cumulant LBM) [17, 18, 19], CLBM (cascaded LBM) [16], ELBM (entropic LBM) [8], or KBC-LBM [24], to give some examples. They vary in the numerical stability mainly with respect to the Reynolds number Re. In this study, the Reynolds number is defined by $\operatorname{Re}=Q_{0} D_{H} /(\nu A)$, where $Q_{0}$ is the volumetric flow rate $(65,91,219$, and $303 \mathrm{ml} / \mathrm{s}), D_{H}$ is the hydraulic diameter $D_{H}=4 A / P, A$ is the cross-sectional area ( $A$ cancels out in the formula for Re), $P$ is the wetted perimeter (given in Table 1 ), and $\nu$ is the kinematic viscosity of the fluid (water in this study with $\nu=10^{-6} \mathrm{~m}^{2} / \mathrm{s}$ ). Reynolds number is a measure of turbulence in a flow. For $\operatorname{Re}<2000$, laminar flow (consisting of individual layers of fluid gliding along each other) is observed, whereas $\operatorname{Re}>4000$ suggests turbu- 
lent flow (individual layers of flowing fluid merge and unstable vortices appear), [12]. For the flow regimes investigated experimentally in this study, the values of Re ranged between 4000 and 35000, see Table 1 . The state-of-the-art CuLBM is known to perform well for turbulent flows [17, 18, 19, 15] and was therefore used for all numerical simulations.

All simulations presented here were carried out on a computational cluster equipped with 12 GPU compute nodes, each with 4 Nvidia Tesla V100 (32 GiB RAM). In order to assure numerical convergence of the results, the simulations were computed on gradually refined grids described in Table 2 and, as illustrated in Figure 3, comparable numerical results were obtained. All results presented in this paper were computed with the final time $T=4 \mathrm{~s}$ on Grid 5 (with $3000 \times 384 \times 384$ lattice sites and spatial resolution $h=68.3 \mu \mathrm{m}$ ) and time step $4 \mu \mathrm{s}$. The computational times are also given in Table 2 .

\subsection{Data averaging and spatial integration}

The PC-MRI experimental data acquisition is performed using $2 \mathrm{D}$ vertical slices perpendicular to the phantom tube. The location and magnitude of the forward and backward flow along the horizontal axis $x$ is determined using the following averaging and spatial integration technique applied both to experimental and simulation data for all $x \in\left[0, L_{x}\right]$, which represents the investigated segment of the flow phantom, as shown in Figure 1 .

First, at every point $(x, y, z)$ of surface $A_{y z}(x)$ of liquid-filled space in the $(y, z)$ plane at $x \in\left[0, L_{x}\right]$, the fluid velocity component $v_{x}$ (parallel to the $x$-axis) is averaged over $N$ samples. The $x$-axis of the model corresponds to the axis of symmetry of the MRI scanner and the phantom tube. In the PC-MRI experiment, $N$ stands for the total number of repeatedly measured images of a particular slice at given position $x$ ( $N$ being 400 and 200 for $1.5 \mathrm{~T}$ and $3 \mathrm{~T}$ field strengths, respectively).

For the simulated results, $N$ denotes the total number of equidistantly distributed samples taken from the time interval $\left[t_{i n i}, t_{f i n}\right]$, with $t_{i n i}=3 \mathrm{~s}$ and $t_{f i n}=4 \mathrm{~s}$ selected as the sufficient time interval. The simulation time step of $4 \mu$ s corresponds to $N=250000$.

Since the values of interest are the positive and negative parts of the fluid flux in the $x$-axis direction (i.e., the forward and backward flows), the notations $v_{x}^{+}=$ $\max \left\{0,\left\langle v_{x}\right\rangle\right\}$ for the positive and $v_{x}^{-}=\min \left\{0,\left\langle v_{x}\right\rangle\right\}$ for the negative velocity parts are used, with $\left\langle v_{x}\right\rangle$ denoting the averaged horizontal fluid velocity component. Then, the values $v_{x}^{+}$and $v_{x}^{-}$are spatially integrated over the surface $A_{y z}(x)$ and the resulting positive and negative fluxes are denoted by $q_{x}^{+}$and $q_{x}^{-}$, respectively. Note that for all $x \in\left[0, L_{x}\right]$, the sum of these fluxes equals to the input flow rate by definition.

The data averaging and subsequent spatial integration technique is employed to reduce the noise produced by the hyposignal acrylic walls during the PC-MRI data acquisition. In the case of CFD, the temporal averaging is one of the tools for describing and characterizing turbulent flows [33].

\subsection{Turbulence intensity}

According to [33], the turbulent flow regime behind the valve can be quantified by the turbulence intensity. It is defined as the root-mean-square of velocity fluctuations divided by the characteristic fluid velocity $v_{0}$ (which is given in Table 1). Then, the turbulent intensity is spatially averaged over $A_{y z}(x)$ for all $x \in\left[0, L_{x}\right]$ and the resulting (dimensionless) quantity is denoted by $T i(x)$.

\subsection{Statistical methods}

To compare the LBM with PC-MRI measurements, a series of Bland-Altman tests [2] was performed. For each experimental slice position, a corresponding slice from the LBM data was selected to provide a set of simulation-experiment data pairs. The quantities subjected to comparison were the forward and backward flows. Their values obtained for each slice were, prior to the Bland-Altman analysis, normalized by their CFDobtained maximal absolute values over all the slices, in order to avoid a potential distortion of the normalized results (caused by the underestimation of experimental data).

A statistical correlation was performed for each corresponding simulation-experiment data pair. In the cases where the Pearson correlation coefficient of the data was statistically significant (with the level of significance $\alpha=0.05$ ), the normality of the distribution of differences was tested by the Kolmogorov-Smirnov test (with the level of significance $\alpha=0.05$ ). If the normality was not rejected, the sample mean of the differences $\underline{d}$ and the sample standard deviation $s$ of the sample of differences were computed. Since the distribution of differences was considered normal and its variance was unknown, the t-statistics were used to compute the limits of the $95 \%$ confidence interval for the mean of the differences as $\underline{d} \pm t \sqrt{s^{2} / n}$, where $n$ is the sample size. The majority of the differences is supposed to lie within $\underline{d} \pm 2 s$. T-statistics show that the $95 \%$ confidence intervals for $\underline{d}-2 s$ and $\underline{d}+2 s$ are $\underline{d}-2 s \pm t \sqrt{3 s^{2} / n}$ and 


\begin{tabular}{cccccc}
\hline Grid id. & Dimensions & Grid size & $\begin{array}{c}\text { Total GPU } \\
\text { memory } \\
{[\mathrm{GiB}]}\end{array}$ & $\begin{array}{c}\text { Computational } \\
\text { time }\end{array}$ & $\begin{array}{c}\text { GPUs used } \\
\text { (using MPI) }\end{array}$ \\
\hline 1 & $1000 \times 128 \times 128$ & $2.07 \cdot 10^{-4}$ & 4.0 & $43 \mathrm{~min}$ & $4 \times$ Nvidia Tesla V100 32GiB RAM \\
2 & $1500 \times 192 \times 192$ & $1.37 \cdot 10^{-4}$ & 13.5 & $2 \mathrm{~h} 24 \mathrm{~min}$ & $4 \times$ Nvidia Tesla V100 32GiB RAM \\
3 & $2000 \times 256 \times 256$ & $1.03 \cdot 10^{-4}$ & 32.0 & $5 \mathrm{~h} 14 \mathrm{~min}$ & $4 \times$ Nvidia Tesla V100 32GiB RAM \\
4 & $2500 \times 320 \times 320$ & $8.21 \cdot 10^{-5}$ & 62.4 & $11 \mathrm{~h} 4 \mathrm{~min}$ & $4 \times$ Nvidia Tesla V100 32GiB RAM \\
5 & $3000 \times 384 \times 384$ & $6.83 \cdot 10^{-5}$ & 107.9 & $20 \mathrm{~h} 47 \mathrm{~min}$ & $4 \times$ Nvidia Tesla V100 32GiB RAM \\
6 & $3750 \times 480 \times 480$ & $5.46 \cdot 10^{-5}$ & 216 & $21 \mathrm{~h} \mathrm{39} \mathrm{min}$ & $8 \times$ Nvidia Tesla V100 32GiB RAM \\
\hline
\end{tabular}

Table 2 Computational grid parameters used in the numerical simulations, total memory needed on GPU (in single precision), and computational time (wall time) to simulate $4 \mathrm{~s}$ of $3 \mathrm{D}$ fluid flow.

$\underline{d}+2 s \pm t \sqrt{3 s^{2} / n}$, respectively. Therefore, according to [2], the interval $\left(\underline{d}-2 s-t \sqrt{3 s^{2} / n}, \underline{d}+2 s+t \sqrt{3 s^{2} / n}\right)$ was chosen as the limits of agreement (LoA).

\section{Results}

\subsection{Forward and backward flow}

For all three valve plates, the experimentally measured and simulated forward and backward flow profiles are shown in Figure 4, and Table 3 summarizes the results of the Bland-Altman data analysis. When the measured forward or backward flows were spatially distorted, the normality of the data was rejected (cf. "NR" in Table 3) or the LoA range was large. Otherwise, the normality allowed obtaining the LoA ranges, which are shown in Table 3 .

Figure 4 demonstrates that the data from the $1.5 \mathrm{~T}$ and $3 \mathrm{~T}$ scanners agree well with each other in the case of Mild-VS valve plate. The discrepancy grows for the moderate and severe stenoses (Mod-VS and Sev-VS), in which the largest disagreement is observed in the experiments with the highest flow rate $(303 \mathrm{ml} / \mathrm{s})$. However, the results of statistical analysis in Table 3 show that no substantial effect of the magnetic field strength is apparent.

As expected, when comparing the experimentally measured values with the simulations, the highest degree of agreement is found in the case of the Mild-VS valve plate, where the LoA remains in the interval of $(-6$ to +10$) \%$ for the forward flow and $(-32$ to +36$) \%$ for the backflow. The mean difference is contained in the interval $(0$ to +5$) \%$ and $(-5$ to +2$) \%$ for the forward and backward flow, respectively. In the Mod-VS case, the agreement is decreasing (see Table 3). Finally, in the Sev-VS case, there is a severe underestimation of the measured flow when compared to LBM with LoA being in the intervals $(-34$ to +20$) \%$ and $(-130$ to +62$) \%$ for the forward and backward flow, respec- tively. The underestimation of MRI measurements is manifested as a negative mean difference in the case of turbulent flow through Sev-VS valve plate. All the aforementioned LoA intervals are symmetric around the mean difference.

\subsection{Turbulence effects}

Figures 5-7 show the difference between the experimentally and computationally obtained backward fluxes, both for $1.5 \mathrm{~T}$ and $3 \mathrm{~T}$ cases, together with the spatially averaged turbulence intensity $T i$. The difference $\left|q_{\text {sim }}^{-}\right|-\left|q_{\text {exp }}^{-}\right|$, evaluated at the positions of the PCMRI slices, quantifies the discrepancy of the backward flow between the simulation and PC-MRI measurement (positive value for the underestimation by PC-MRI).

At the position of around $4.5 \mathrm{~cm}$ behind the valve, the underestimation reaches its maximum value, after which it progressively decreases and reaches its minimum value $7-8 \mathrm{~cm}$ behind the valve. In the closest vicinity of the valve (at a distance of less than $2 \mathrm{~cm}$ from the valve), we observe some level of overestimation. At the position of approximately $2-3 \mathrm{~cm}$ behind the valve, the discrepancy between the simulated and measured backward flow is minimal. The onset of turbulence precedes the maximum PC-MRI underestimation. The peak turbulence intensity is at around $3 \mathrm{~cm}$ behind the valve, after which it slowly decreases.

\section{Discussion}

The PC-MRI experimental measurements of flow through a custom-designed in vitro aortic valve phantom, which were performed on $1.5 \mathrm{~T}$ and $3 \mathrm{~T}$ MRI scanners, were compared to the results of a 3D LBM numerical model. The data was analyzed using averaging and integration techniques to obtain forward and backward fluxes 


\begin{tabular}{ccccccc}
\hline \multicolumn{3}{c}{ Mild-VS } & \multicolumn{2}{c}{ Mod-VS } & \multicolumn{2}{c}{ Sev-VS } \\
$B_{0}$ & LoA & MD & LoA & MD & LoA & MD \\
& range & & range & & range & \\
{$[T]$} & {$[\%]$} & {$[\%]$} & {$[\%]$} & {$[\%]$} & {$[\%]$} & {$[\%]$} \\
\hline
\end{tabular}

Flow rate $65 \mathrm{ml} / \mathrm{s}$ :

\begin{tabular}{|c|c|c|c|c|c|c|}
\hline 1.5 & $(0,10)$ & 5 & $(-10,14)$ & 2 & $(-24,20)$ & -2 \\
\hline ? & $(-3,5)$ & 1 & $(-10,20)$ & 5 & $(-24,20)$ & -2 \\
\hline \multicolumn{7}{|c|}{ Flow rate $91 \mathrm{ml} / \mathrm{s}$ : } \\
\hline 1.5 & NR & & $(-6,9)$ & 1 & $(-25,14)$ & -5 \\
\hline & $(-5,7)$ & 1 & $(-5,10)$ & 2 & $(-28,12)$ & -8 \\
\hline \multicolumn{7}{|c|}{ Flow rate $129 \mathrm{ml} / \mathrm{s}$ : } \\
\hline 1.5 & $(-1,5)$ & 2 & $(-7,7)$ & 0 & $(-22,12)$ & -5 \\
\hline & $(-3,5)$ & 1 & $(-6,11)$ & 3 & $(-34,13)$ & -11 \\
\hline
\end{tabular}

Flow rate $303 \mathrm{ml} / \mathrm{s}$ :

$\begin{array}{lllllll}1.5 & (-5,6) & 1 & (-11,8) & -2 & (-26,6) & -10\end{array}$

$3 \quad(-6,5) \quad 0 \quad(-10,10) \quad 0 \quad(-25,12) \quad-6$

\section{Backward flow}

Flow rate $65 \mathrm{ml} / \mathrm{s}$ :

$$
\begin{array}{llllll}
1.5 & \mathrm{NR} & (-69,68) & -1 & (-94,57) & -18
\end{array}
$$$$
3 \quad(-27,32) \quad 2 \quad(-71,83) \quad 6 \quad(-95,62) \quad-17
$$

Flow rate $91 \mathrm{ml} / \mathrm{s}$ :

$\begin{array}{llllll}1.5 & \mathrm{NR} & (-35,40) & 3 & (-86,40) & -23\end{array}$

$3 \quad(-32,36) \quad 2 \quad(-40,55) \quad 8 \quad(-109,43) \quad-33$

Flow rate $129 \mathrm{ml} / \mathrm{s}$ :

$1.5(-15,13) \quad-1(-38,37) \quad-1 \quad(-94,42) \quad-26$

$3(-26,16) \quad-5(-36,46) \quad 5 \quad(-130,53) \quad-39$

Flow rate $303 \mathrm{ml} / \mathrm{s}$ :

$1.5(-21,18) \quad-2(-61,36) \quad-12(-83,39) \quad-22$

$3(-28,20) \quad-4(-48,27) \quad-11(-75,33) \quad-21$

$\mathrm{NR}=$ normality rejected by Kolmogorov-Smirnov test

LoA $=$ Limit of agreement

$\mathrm{MD}=$ Mean difference

Table 3 Limit of agreement (LoA) range $\left(\mathrm{LoA}^{-}, \mathrm{LoA}^{+}\right)$ (in \%) of the Bland-Altman comparison of simulated and experimental data. In each case (type of valve, flow rate and magnetic field), a lower $\left(\mathrm{LoA}^{-}\right)$and higher $\left(\mathrm{LoA}^{+}\right)$limit of agreement is presented. The mean difference (MD) lies in the middle of each interval $\left(\mathrm{LoA}^{-}, \mathrm{LoA}^{+}\right)$.

through the slices along the horizontal axis of the flowphantom up to $8 \mathrm{~cm}$ behind the valve. The LBM used in this work has been validated in a number of studies $[21,9,17,18,19,16,8,24,14,35,34]$ and the inputs for the model are solely the geometry and the measured inlet flow. Hence, here, the model can be considered as an "in silico" gold standard, which is then used to explain the effect of turbulence on the underestimation of backward flow measured by PC-MRI.

The simulated and measured results of forward and backward flows show an excellent agreement for MildVS with the discrepancy between simulated and mea- sured fluxes (both forward and backward) being below $5 \mathrm{ml} / \mathrm{s}$. However, a large discrepancy occurred in the case of the severely stenotic valve plate (Sev-VS). This can be explained by the signal void in the voxels in which a rapid turbulent vortex assembles a population of spins with different encoded phases during the readout period. The measured phase distribution is therefore broadened due to a decreased signal-to-noise ratio (SNR) and, thus, the average phase in the voxel is underestimated. We remark that the evidence for the signal void was observed in the magnitude images.

The backward flow was shown to be more prone to the underestimation than the forward flow. This can be caused by a lower velocity magnitude of the backward flow with respect to the forward flow. Slower spins spend more time in an excited slice and are, therefore, subject to multiple excitation, resulting in the lower transverse magnetization and, consequently, a decreased SNR. In addition, higher flow rates tend to lead to higher simulation-data discrepancies than lower flow rates, which can be explained by the increased level of turbulence. Indeed, the turbulence intensity has a well visible peak when its value is above 0.10 (cf. the highest flow rate in Figures $5 \mathrm{~d}, 6 \mathrm{~d}$, and $7 \mathrm{~d}$, or the Sev-VS in Figures $7 \mathrm{~b}, 7 \mathrm{c}$, and $7 \mathrm{~d}$ ). On the contrary, the laminar or low turbulent flow is associated with a rather flat turbulent intensity (cf. Figures 5 and 6a-c), and the level of discrepancy between the simulation and measurement is low.

At the immediate vicinity of the valve plate, some overestimation of the measurement was observed. It is either substantially lower than the significant underestimation further behind the valve (typically in the SevVS valve plate), or present directly on the valve due to an artifact caused by the hyposignal plate material (Mod-VS). Therefore, any direct conclusion cannot be made in the closest vicinity of the valve.

We remark that when considering extending the model to a more realistic scenario involving non-rigid geometry, the advantage of using LBM is that any change in internal geometry does not involve a time consuming re-meshing of the computational domain, which is typically the case for most CFD methods. Additionally, our preliminary simulations of time-varying flow indicate that the computational intensity is of the same order as for the simplified situation presented in this paper.

The model implementation in this work uses an inhouse $\mathrm{C}++$ and CUDA computer code that can be executed efficiently on massively parallel hardware such as GPUs. Due to this, the CFD model is solved within tens of minutes to a few hours depending on the spatiotemporal resolution and the exact type of GPU used. 
The ongoing work of our group on the development of efficient computational methods and code optimization will allow a further acceleration of these computations. However, even the current "proof-of-concept" work demonstrates that our modeling study can be performed within the time window between the acquisition of cardiovascular MRI and the complete clinical analysis. Therefore, it can provide extra information, such as turbulence quantification, in clinically relevant time and have the potential to increase the accuracy of the measurement thanks to combining a physical model and measured data [5].

\subsection{Perspectives of the work}

In addition to the main objective of the presented work, i.e., employing a CFD model to investigate and quantify the PC-MRI flow underestimation due to the presence of turbulent flow, we will describe some possible perspectives of incorporating the models in the workflow of a PC-MRI exam, which could be derived from the present study.

In turbulent regimes, a single clinical measurement of instantaneous velocity profile at a given spatio-temporal point cannot provide relevant flow characteristics, such as the mean velocity and its variance (i.e., the turbulence intensity) to assess the level of PC-MRI flow underestimation. To get a statistically significant set of data, the time averaging technique requires the number of repeated acquisitions in the order of tens, which is highly time-demanding and, therefore, not acceptable in clinical workflow. However, a CFD model used in the post-processing phase can fill that gap. The model, when adjusted to measured PC-MRI, can provide a large number of velocity profiles of the same turbulent flow. Therefore, a detailed assessment of turbulent flow can be performed (as is usual, for instance, in aeronautics) using the mean velocity, standard deviation, intensity of turbulence, skewness, kurtosis, etc. [33].

The location of the maximum turbulence intensity and of the highest experiment-simulation discrepancy can contribute to optimize the measurement. In the future, we can anticipate a fast CFD model suggesting the optimal positioning of the measurement slice, prior to performing a high-fidelity $2 \mathrm{D}$-flow measurement. The superior spatio-temporal resolution or fast acquistions may be crucial (and advantageous even to 4D flow acquisitions) in some applications, e.g., for flow measurement under pharmacological or exercise stress [32, 31]. Indeed, the highest flow rate in our experiment corresponds to the situation of increased cardiac output. The flow measurement by PC-MRI is known to be very challenging under such conditions but has the important clinical potential to characterize especially earlier stages of some diseases or assess early-stage heart failure. Coupling of the data of suboptimal quality with a CFD model would therefore be beneficial.

\subsection{Study limitations}

Our study was limited to non-pulsatile flow through a rigid tube of simple geometry. Even though the nonpulsatile flow is a significant simplification to the in vivo scenario, its use made it possible to acquire a statistically significant amount of data.

The material properties of the wall did not match the properties of tissue in the cardiovascular system. We therefore could not completely assess the influence of tissues surrounding aorta in vivo on the patient examination. However, this simplification allowed to employ a robust CFD model, which is well validated in such a setup (i.e., when having only inlet flow rate and the geometry as the inputs) and thanks to this, directly derive conclusions about the PC-MRI measurements.

Another simplification was replacing water instead of blood or a blood-like fluid. Prior to using more complex phantoms or coupling our model with in vivo data, the presented work aims at making the initial step in a simplified regime under the assumption of Newtonian fluid flow. When extending our phantom by using blood-like fluid, more complex fluid descriptions will be investigated, including non-Newtonian regimes.

\section{Conclusions}

The presented work shows that the backward flow can be measured by PC-MRI accurately under laminar or low turbulent conditions. However, a high level of turbulence leads to an underestimated backward flow as indicated by the spatial distribution of the turbulence intensity and the difference between the measured and simulated backward fluxes.

The results of this paper, even though presented using a simplified setup of a phantom experiment, demonstrate the future vision of estimating the measurement error, quantification of the level of turbulence or optimal positioning of the measuring plane. The presented paper is an essential initial step towards combining acquired data by PC-MRI with CFD model suitable for the use in clinical workflow, allowing to enhance the interpretation of measured data by a biophysical model. This has great potential to play a substantial role in some non-resolved problems, such as, for instance, the 
aortic valve stenosis grading [13], particularly in the moderate to severe lesions.

Acknowledgements We would like to acknowledge Dr T. Hussain (UT Southwestern Medical Center Dallas, USA) and A. Wodecki (Czech Technical University in Prague) for valuable discussions.

\section{Conflict of interest}

The authors declare that they have no conflict of interest.

\section{Authors' contribution statement}

- R. Fučík: Study conception and design, analysis and interpretation of data, drafting of manuscript, critical revision.

- R. Galabov: Study conception and design, acquisition of data, analysis and interpretation of data, drafting of manuscript.

- P. Pauš: Analysis and interpretation of data, drafting of manuscript, critical revision.

- P. Eichler: Acquisition of data, analysis and interpretation of data, critical revision.

- J. Klinkovský: Acquisition of data, analysis and interpretation of data, critical revision.

- R. Straka: Study conception and design, analysis and interpretation of data, drafting of manuscript critical revision.

- J. Tintěra: Study conception and design, critical revision.

- R. Chabiniok: Study conception and design, analysis and interpretation of data, drafting of manuscript, critical revision.

\section{References}

1. Anderson JR, Diaz O, Klucznik R, Zhang YJ, Britz GW, Grossman RG, Lv N, Huang Q, Karmonik C (2014) Validation of computational fluid dynamics methods with anatomically exact, $3 \mathrm{D}$ printed MRI phantoms and 4D pcMRI. In: 2014 36th Annual International Conference of the IEEE Engineering in Medicine and Biology Society, IEEE, pp 66996701, DOI 10.1109/EMBC.2014.6945165

2. Bland JM, Altman D (1986) Statistical methods for assessing agreement between two methods of clinical measurement. The lancet 327(8476):307-310

3. Caiazzo A, Junk M (2008) Boundary forces in lattice Boltzmann: Analysis of momentum exchange algorithm. Computers \& Mathematics with Applications 55(7):1415-1423, DOI 10.1016/j.camwa.2007.08.004

4. Chai P, Mohiaddin R (2005) Slice location dependence of aortic regurgitation measurements with MR phase velocity mapping. J Cardiovasc Magn Reson 7(4):705-716, DOI 10.1081/JCMR-65639

5. Chapelle D, Fragu M, Mallet V, Moireau P (2013) Fundamental principles of data assimilation underlying the Verdandi library: applications to biophysical model personalization within euHeart. Medical \& biological engineering \& computing 51(11):12211233, DOI 10.1007/s11517-012-0969-6

6. Chaturvedi A, Hamilton-Craig C, Cawley PJ, Mitsumori LM, Otto CM, Maki JH (2016) Quantitating aortic regurgitation by cardiovascular magnetic resonance: significant variations due to slice location and breath holding. Eur Radiol 26(9):31803189, DOI 10.1007/s00330-015-4120-6

7. Chatzimavroudis GP, Walker PG, Oshinski JN, Franch RH, Pettigrew RI, Yoganathan AP (1997) Slice location dependence of aortic regurgitation measurements with MR phase velocity mapping. Magn Reson Med 37(4):545-551, DOI 10.1002/mrm.1910370412

8. Chikatamarla S, Ansumali S, Karlin IV (2006) Entropic lattice Boltzmann models for hydrodynamics in three dimensions. Physical review letters 97(1):010201, DOI 10.1103/PhysRevLett.97.010201

9. d'Humieres D (2002) Multiple-relaxation-time lattice Boltzmann models in three dimensions. Philosophical Transactions of the Royal Society of London Series A: Mathematical, Physical and Engineering Sciences 360(1792):437-451, DOI 10.1098/rsta.2001.0955

10. Donati F, Myerson S, Bissell MM, Smith NP, Neubauer S, Monaghan MJ, Nordsletten DA, Lamata P (2017) Beyond Bernoulli: Improving the Accuracy and Precision of Noninvasive Estimation of Peak Pressure Drops. Circ Cardiovasc Imaging 10(1):e005207, DOI 10.1161/CIRCIMAGING.116.005207

11. Dyverfeldt P, Bissell M, Barker AJ, Bolger AF, Carlhäll CJ, Ebbers T, Francios CJ, Frydrychowicz A, Geiger J, Giese D, et al. (2015) 4D flow cardiovascular magnetic resonance consensus statement. J Cardiovasc Magn Reson 17(1):72, DOI 10.1186/s12968-015-0174-5

12. Eckhardt B (2008) Introduction. Turbulence transition in pipe flow: 125th anniversary of the publication of Reynolds' paper. DOI $10.1098 /$ rsta. 2008.0217 
13. Everett RJ, Clavel MA, Pibarot P, Dweck MR (2018) Timing of intervention in aortic stenosis: a review of current and future strategies. Heart 104(24):2067-2076, DOI 10.1136/heartjnl2017-312304

14. Fučík R, Eichler P, Straka R, Pauš P, Klinkovský J, Oberhuber T (2019) On optimal node spacing for immersed boundary-lattice Boltzmann method in 2D and 3D. Computers \& Mathematics with Applications 77(4):1144-1162, DOI 10.1016/j.camwa.2018.10.045

15. Gehrke M, Banari A, Rung T (2020) Performance of under-resolved, model-free LBM simulations in turbulent shear flows. In: Hoarau Y, Peng SH, Schwamborn D, Revell A, Mockett C (eds) Progress in Hybrid RANS-LES Modelling, Springer International Publishing, Cham, pp 3-18

16. Geier M, Greiner A, Korvink JG (2006) Cascaded digital lattice Boltzmann automata for high Reynolds number flow. Physical Review E 73(6):066705, DOI 10.1103/PhysRevE.73.066705

17. Geier M, Schönherr M, Pasquali A, Krafczyk M (2015) The cumulant lattice Boltzmann equation in three dimensions: Theory and validation. Computers \& Mathematics with Applications 70(4):507547, DOI 10.1016/j.camwa.2015.05.001

18. Geier M, Pasquali A, Schönherr M (2017) Parametrization of the cumulant lattice Boltzmann method for fourth order accurate diffusion part I: Derivation and validation. Journal of Computational Physics 348:862-888, DOI 10.1016/j.jcp.2017.05.040

19. Geier M, Pasquali A, Schönherr M (2017) Parametrization of the cumulant lattice Boltzmann method for fourth order accurate diffusion Part II: Application to flow around a sphere at drag crisis. Journal of Computational Physics 348:889-898, DOI 10.1016/j.jcp.2017.07.004

20. Goubergrits L, Riesenkampff E, Yevtushenko P, Schaller J, Kertzscher U, Berger F, Kuehne T (2015) Is MRI-Based CFD Able to Improve Clinical Treatment of Coarctations of Aorta? Ann Biomed Eng 43(1):168-176, DOI 10.1007/s10439-014-11163

21. Guo Z, Shu C (2013) Lattice Boltzmann method and its applications in engineering, vol 3. World Scientific

22. Ha H, Lantz J, Ziegler M, Casas B, Karlsson M, Dyverfeldt P, Ebbers T (2017) Evaluation of aortic regurgitation with cardiac magnetic resonance imaging: a systematic review. Sci Rep 7:46618, DOI $10.1038 /$ srep46618
23. Iwamoto Y, Inage A, Tomlinson G, Lee KJ, GrosseWortmann L, Seed M, Wan A, Yoo SJ (2014) Direct measurement of aortic regurgitation with phasecontrast magnetic resonance is inaccurate: proposal of an alternative method of quantification. Pediatr Radiol 44(11):1358-1369, DOI 10.1007/s00247-0143017-x

24. Karlin IV, Bösch F, Chikatamarla S (2014) Gibbs' principle for the lattice-kinetic theory of fluid dynamics. Physical Review E 90(3):031302, DOI 10.1103/PhysRevE.90.031302

25. Kweon J, Yang DH, Kim GB, Kim N, Paek M, Stalder AF, Greiser A, Kim YH (2016) Fourdimensional flow MRI for evaluation of poststenotic turbulent flow in a phantom: comparison with flowmeter and computational fluid dynamics. Eur Radiol 26(10):3588-3597, DOI 10.1007/s00330015-4181-6

26. Lee JC, Branch KR, Hamilton-Craig C, Krieger EV (2018) Evaluation of aortic regurgitation with cardiac magnetic resonance imaging: a systematic review. Heart 104(2):103-110, DOI 10.1136/heartjnl2016-310819

27. Miyazaki S, Itatani K, Furusawa T, Nishino T, Sugiyama M, Takehara Y, Yasukochi S (2017) Validation of numerical simulation methods in aortic arch using 4D Flow MRI. Heart Vessels 32(8):10321044, DOI 10.1007/s00380-017-0979-2

28. Morris PD, Narracott A, von Tengg-Kobligk H, Soto DAS, Hsiao S, Lungu A, Evans P, Bressloff NW, Lawford PV, Hose DR, et al. (2016) Computational fluid dynamics modelling in cardiovascular medicine. Heart 102(1):18-28, DOI 10.1136/heartjnl-2015-308044

29. Nayak KS, Nielsen JF, Bernstein MA, Markl M, Gatehouse PD, Botnar RM, Saloner D, Lorenz C, Wen H, Hu BS, et al. (2015) Cardiovascular magnetic resonance phase contrast imaging. J Cardiovasc Magn Reson 17(1):71, DOI 10.1186/s12968015-0172-7

30. O'Brien KR, Cowan BR, Jain M, Stewart RA, Kerr AJ, Young AA (2008) MRI Phase Contrast Velocity and Flow Errors in Turbulent Stenotic Jets. J Magn Reson Imaging 28(1):210-218, DOI 10.1002/jmri.21395

31. Ruijsink B, Puyol-Antón E, Usman M, van Amerom J, Duong P, Forte MNV, Pushparajah K, Frigiola A, Nordsletten DA, King AP, et al. (2017) Semi-automatic cardiac and respiratory gated MRI for cardiac assessment during exercise. In: Molecular Imaging, Reconstruction and Analysis of Moving Body Organs, and Stroke Imaging and Treatment, Springer, pp 86-95 
32. Ruijsink B, Zugaj K, Pushparajah K, Chabiniok R (2019) Model-based indices of early-stage cardiovascular failure and its therapeutic management in Fontan patients. In: Coudière Y, Ozenne V, Vigmond E, Zemzemi N (eds) Functional Imaging and Modeling of the Heart, Springer International Publishing, Cham, pp 379-387

33. Schlichting H, Gersten K (2016) Boundary-layer theory. Springer

34. Sharma KV, Straka R, Tavares FW (2017) New Cascaded Thermal Lattice Boltzmann Method for simulations of advection-diffusion and convective heat transfer. International Journal of Thermal Sciences 118:259-277, DOI 10.1016/j.ijthermalsci.2017.04.020

35. Sharma KV, Straka R, Tavares FW (2019) Lattice Boltzmann Methods for Industrial Applications. Industrial \& Engineering Chemistry Research 58(36):16205-16234, DOI 10.1021/acs.iecr.9b02008

36. Shen X, Schnell S, Barker AJ, Suwa K, Tashakkor L, Jarvis K, Carr JC, Collins JD, Prabhakaran S, Markl M (2018) Voxel-by-voxel 4D flow MRI-based assessment of regional reverse flow in the aorta. J Magn Reson Imaging 47(5):1276-1286, DOI 10.1002/jmri.25862

37. Sotelo J, Dux-Santoy L, Guala A, RodríguezPalomares J, Evangelista A, Sing-Long C, Urbina J, Mura J, Hurtado DE, Uribe S (2018) 3D axial and circumferential wall shear stress from $4 \mathrm{D}$ flow MRI data using a finite element method and a laplacian approach. Magn Reson Med 79(5):2816-2823, DOI 10.1002/mrm. 26927

38. Srichai MB, Lim RP, Wong S, Lee VS (2009) Cardiovascular applications of phase-contrast MRI. Am J Roentgenol 192(3):662-675, DOI 10.2214/AJR.07.3744

39. Švihlová H, Hron J, Málek J, Rajagopal K, Rajagopal K (2016) Determination of pressure data from velocity data with a view toward its application in cardiovascular mechanics. Part 1. Theoretical considerations. Int J of Eng Sci 105:108-127, DOI 10.1016/j.ijengsci.2015.11.002

40. Švihlová H, Hron J, Málek J, Rajagopal K, Rajagopal K (2017) Determination of pressure data from velocity data with a view towards its application in cardiovascular mechanics. Part 2: A study of aortic valve stenosis. Int J of Eng Sci 114:1-15, DOI 10.1016/j.ijengsci.2017.01.002

41. Wendell DC, Samyn MM, Cava JR, Krolikowski MM, LaDisa JF (2016) The Impact of Cardiac Motion on Aortic Valve Flow Used in Computational Simulations of the Thoracic Aorta. J Biomech Eng 138(9):091001, DOI 10.1115/1.4033964 
a

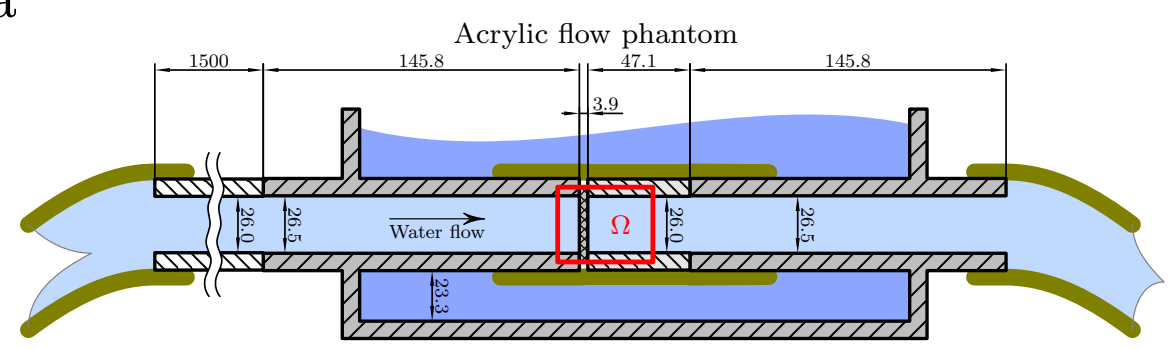

$\mathrm{b}$

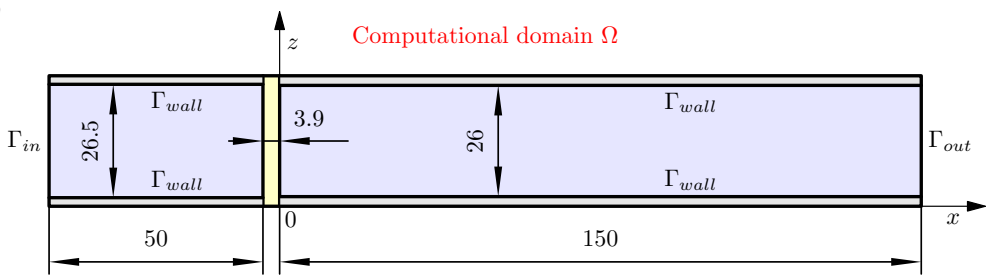

Fig. 1 Depiction of the acrylic flow phantom (a) and the computational domain $\Omega$ (b); vertical slices at $y=0$. All dimensions are in millimeters.

Mild-VS

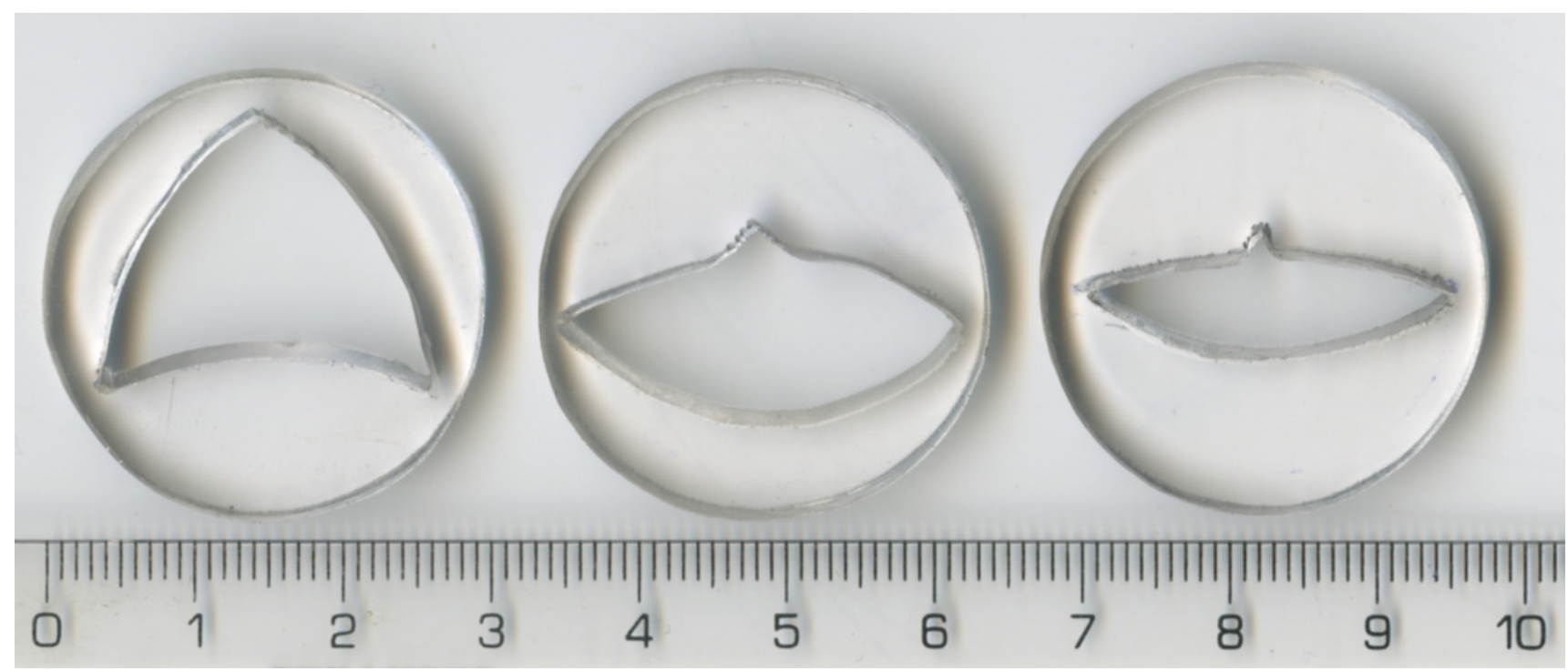

Fig. 2 Three acrylic plates used in the MRI experiments mimicking mild (Mild-VS), moderate (Mod-VS), and severe (Sev-VS) valvular stenosis. The numbers on the ruler represent centimeters $(\mathrm{cm})$. 
a

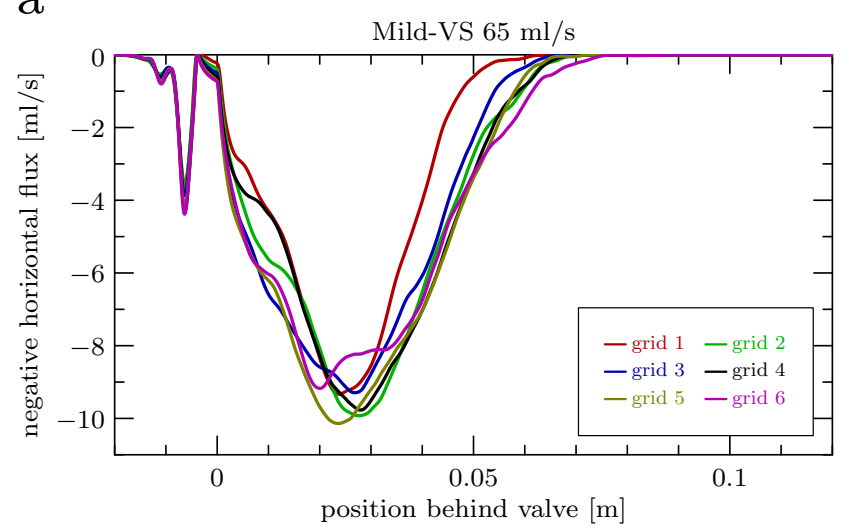

C

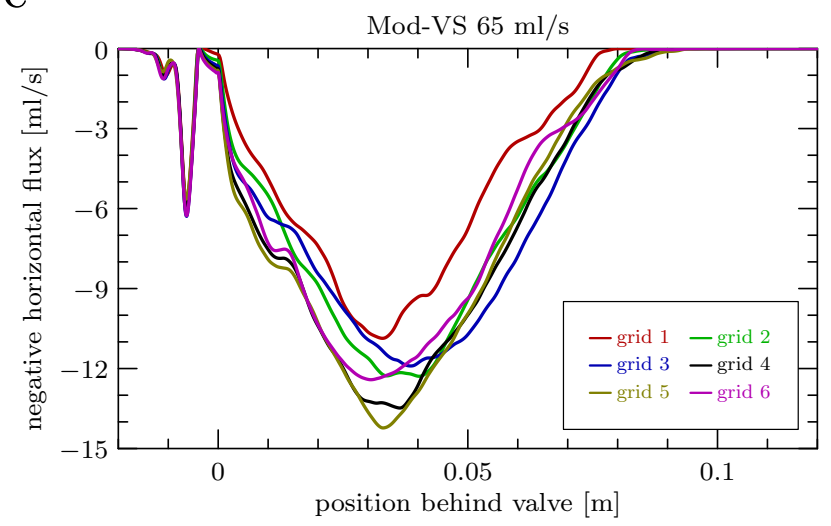

e

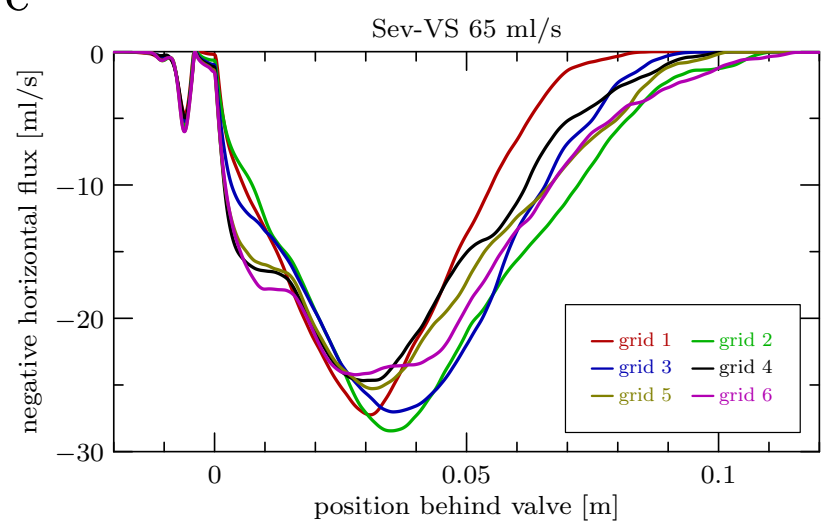

$\mathrm{b}$

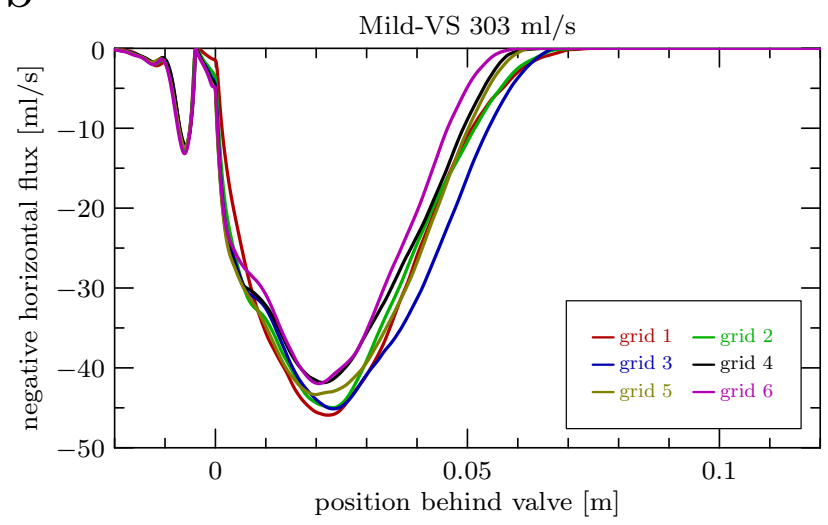

d

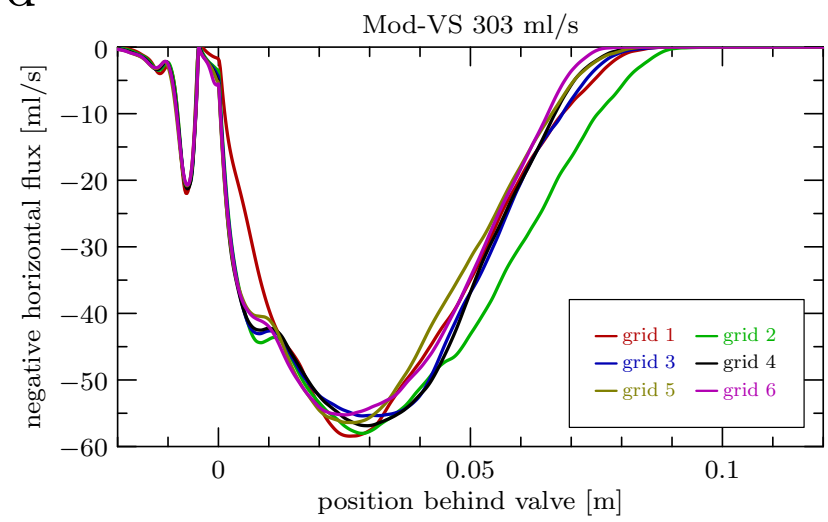

f

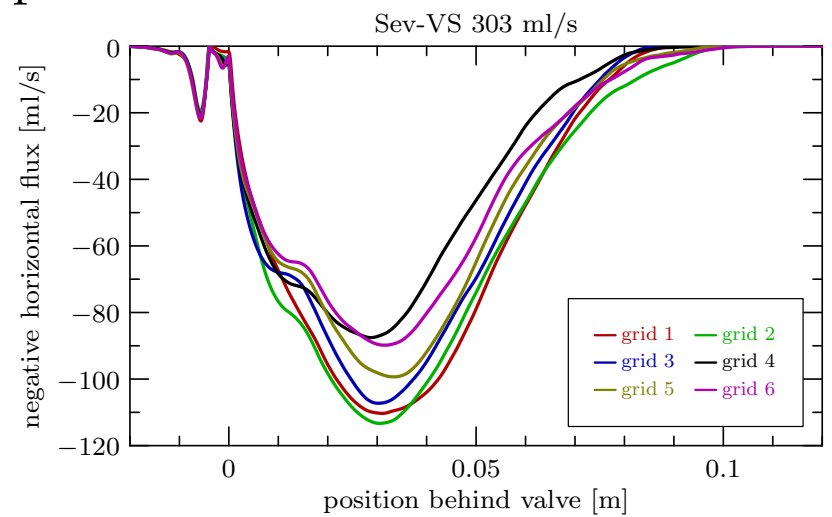

Fig. 3 Grid resolution effects on the simulated backward fluxes for all three valves (Mild-VS, Mod-VS, and Sev-VS) and the lowest ( $\mathrm{a}, \mathrm{c}, \mathrm{e})$ and highest $(\mathrm{b}, \mathrm{d}, \mathrm{f})$ flow rates. The grid properties are given in Table 2 . 
a

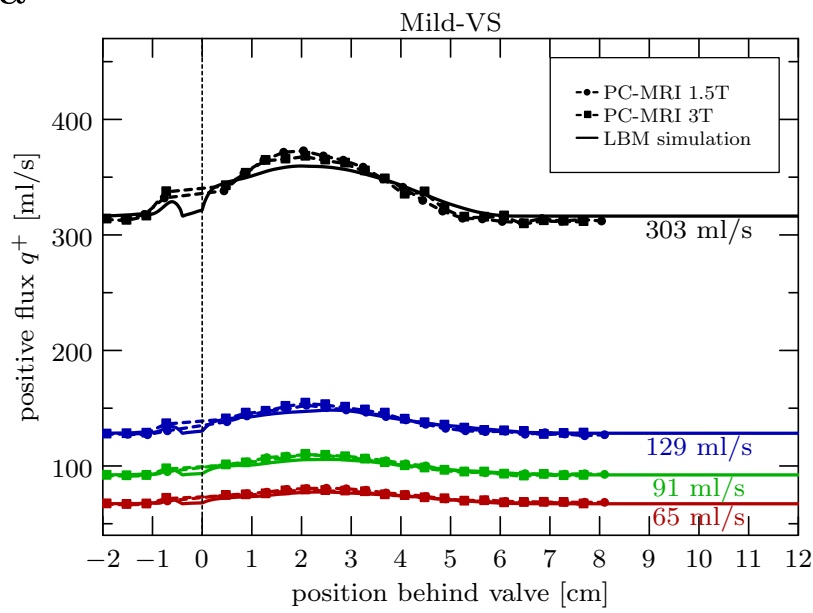

C

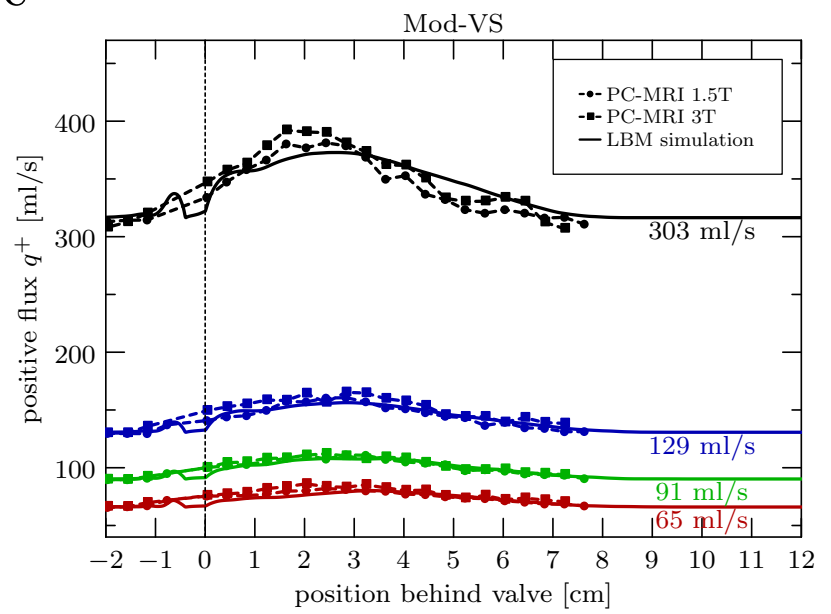

e

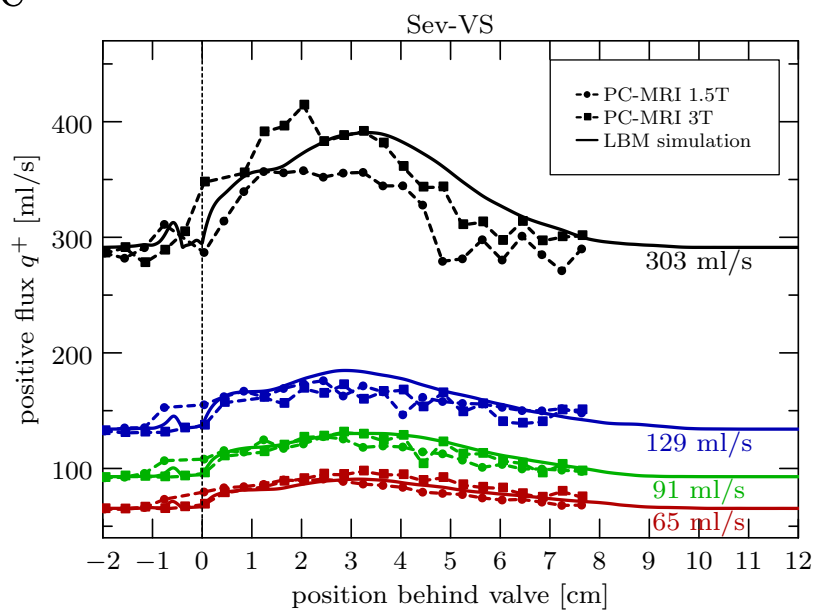

b

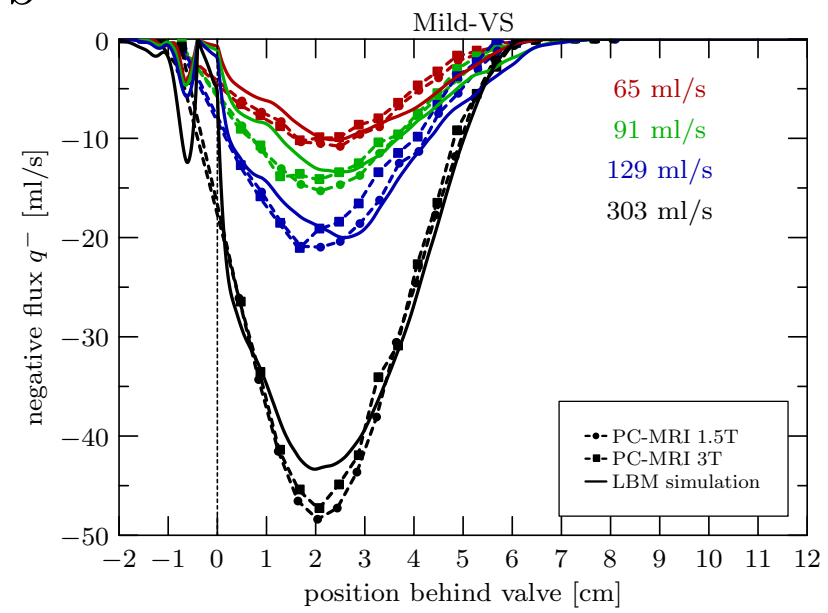

d

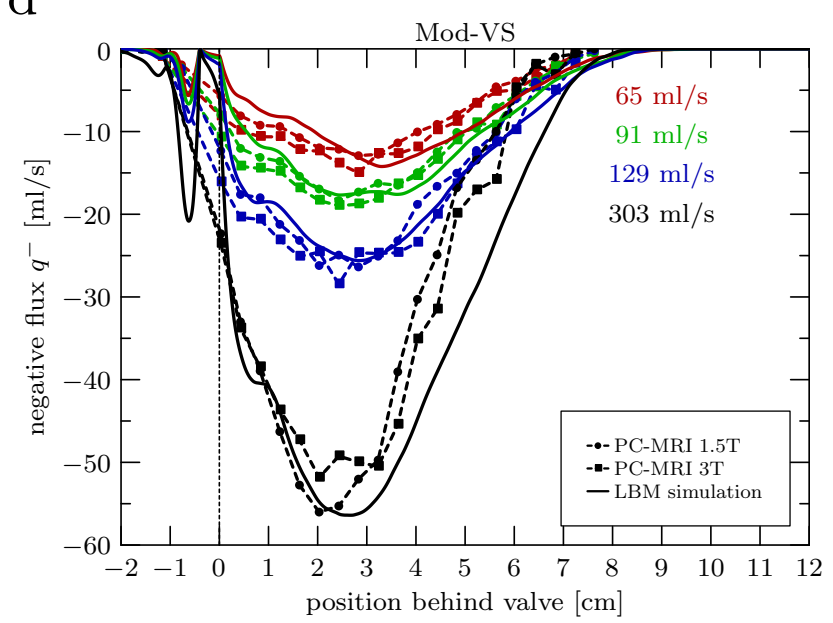

f

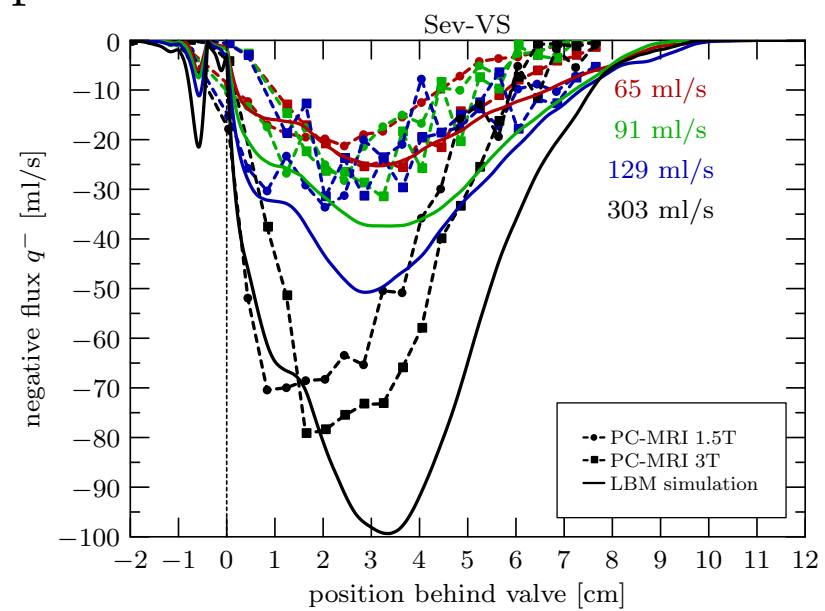

Fig. 4 Forward (a, c, e) and backward (b, d, f) flow profiles for all three valves (mildly, moderately, and severely stenosed, i.e. Mild-, Mod-, Sev-VS, respectively) and all four flow rates that compare the experimental (1.5T and 3T) and LBM-simulated data. The dotted vertical line represents the valve position 
a

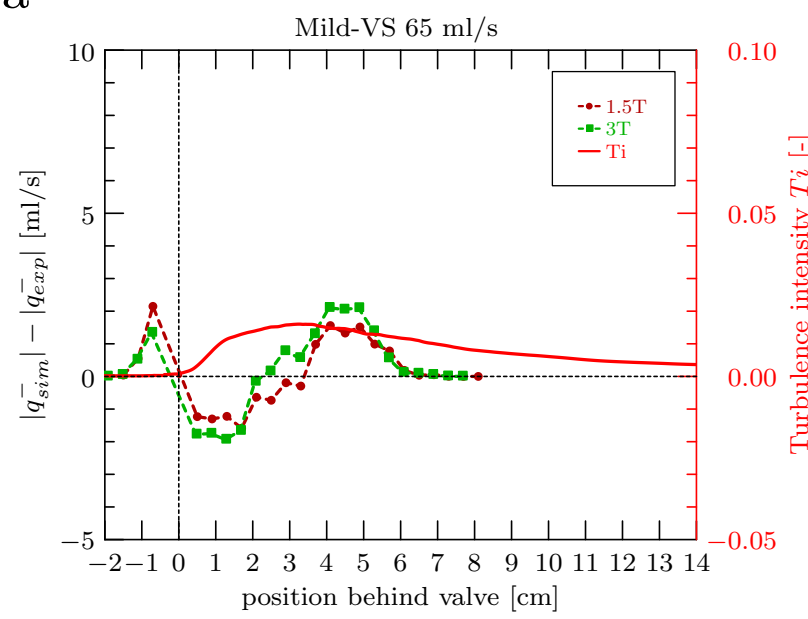

C

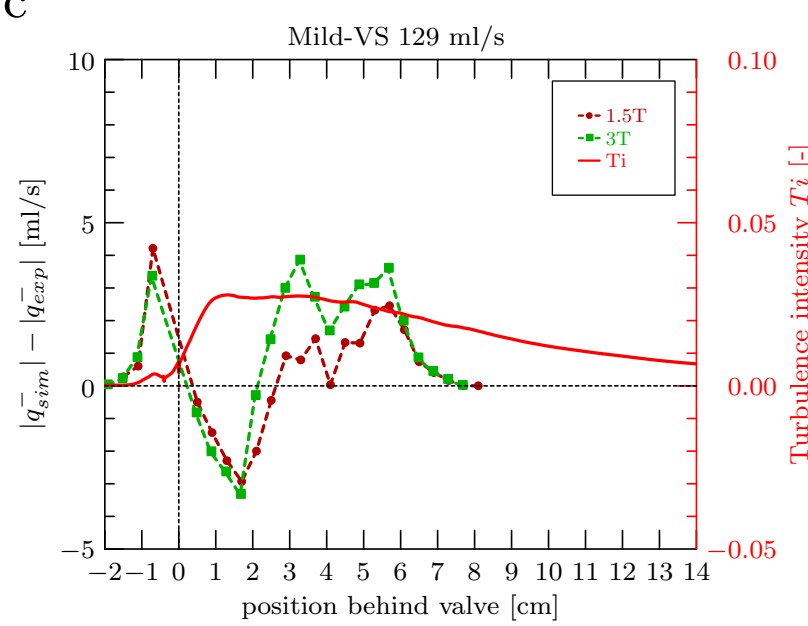

$\mathrm{b}$

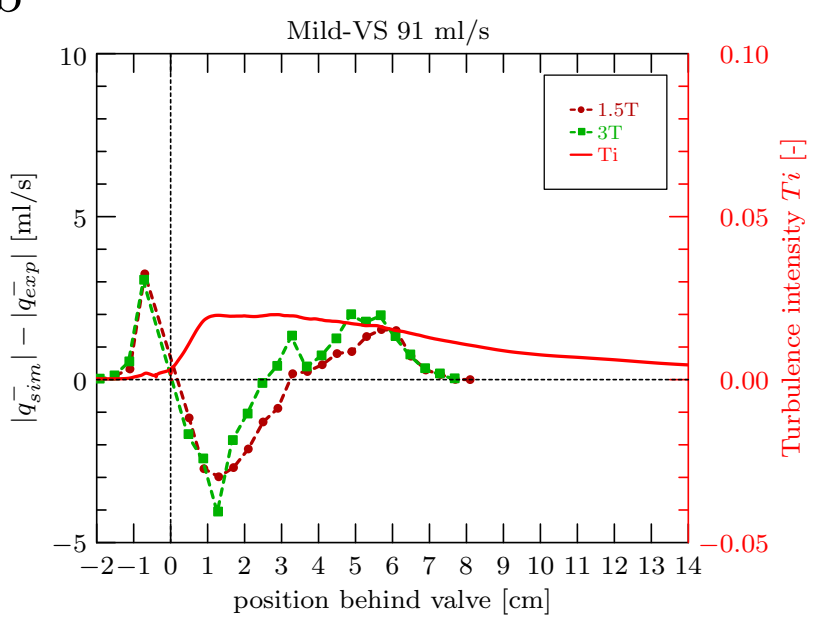

d

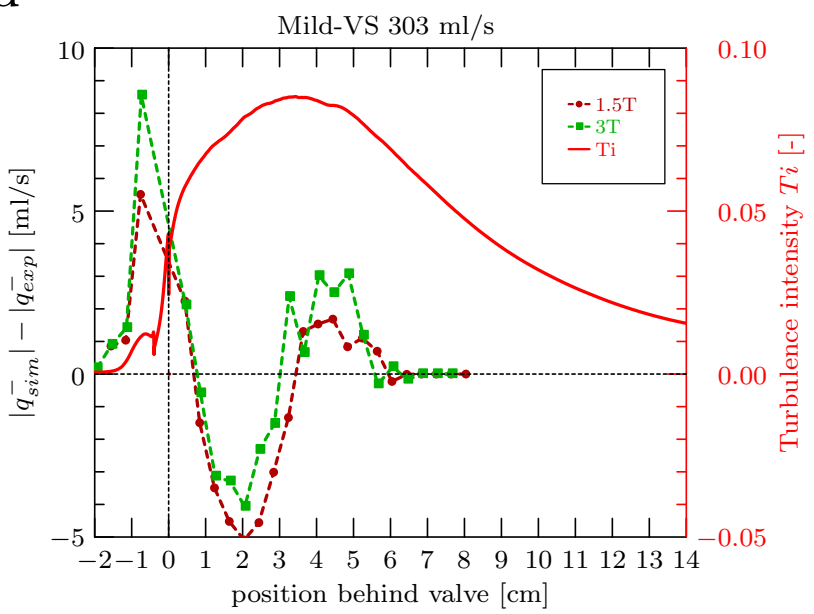

Fig. 5 Horizontal profiles of the difference between experimentally (PC-MRI) and computationally (LBM) obtained magnitudes of backward fluxes $\left|q_{\text {exp }}^{-}\right|$and $\left|q_{s i m}^{-}\right|$, respectively, together with the spatially averaged turbulence intensity for all four flow rates for the mildly stenosed (Mild-VS) valve. The dotted vertical line represents the valve position. 
a

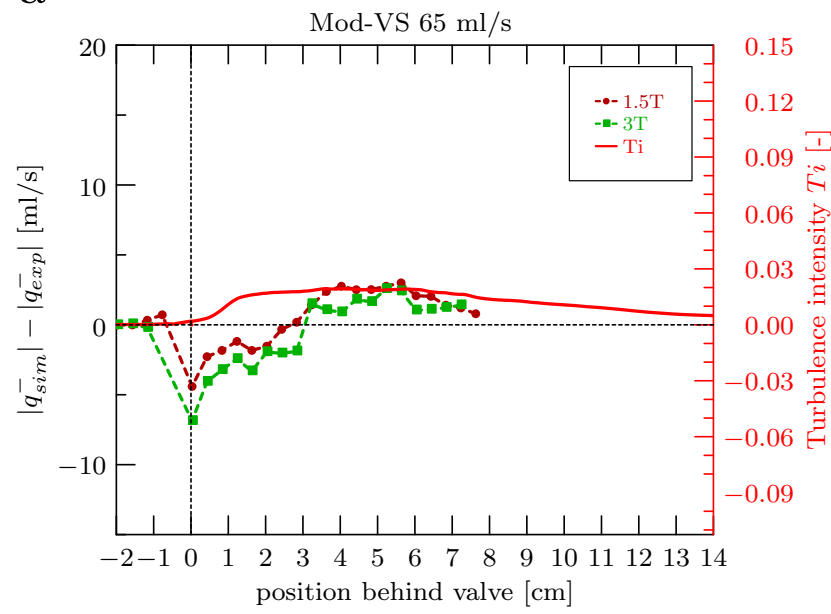

$\mathrm{C}$

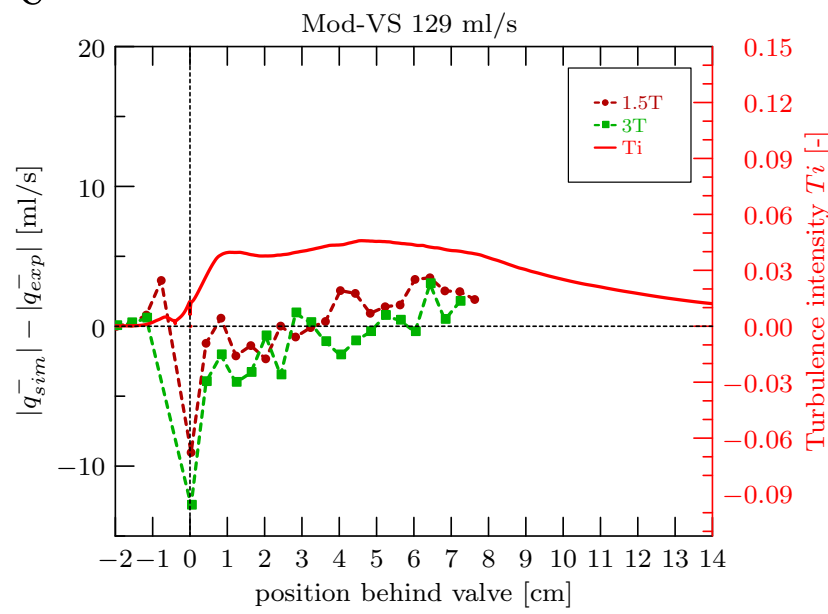

$\mathrm{b}$

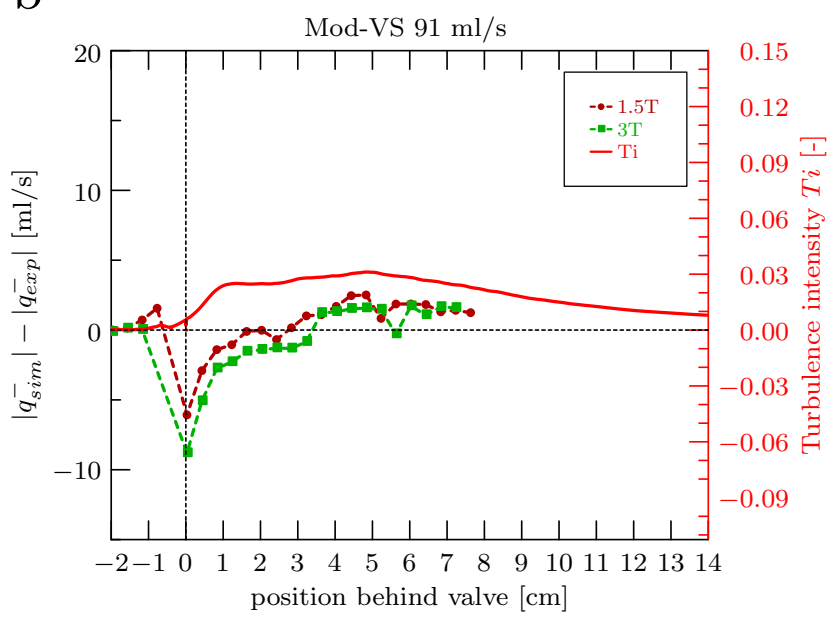

d

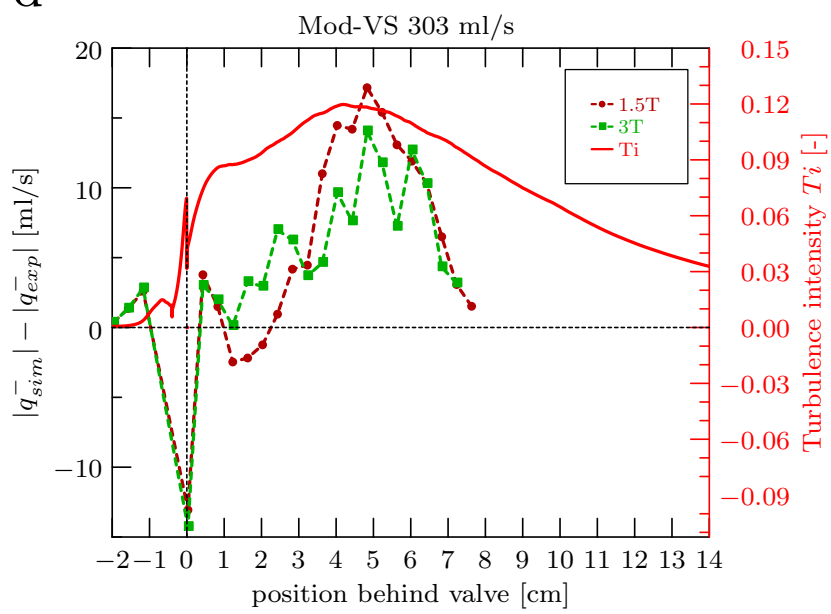

Fig. 6 Horizontal profiles of the difference between experimentally (PC-MRI) and computationally (LBM) obtained magnitudes of backward fluxes $\left|q_{\text {exp }}^{-}\right|$and $\left|q_{s i m}^{-}\right|$, respectively, together with the spatially averaged turbulence intensity for all four flow rates for the moderately-stenosed (Mod-VS) valve. The dotted vertical line represents the valve position. 
a

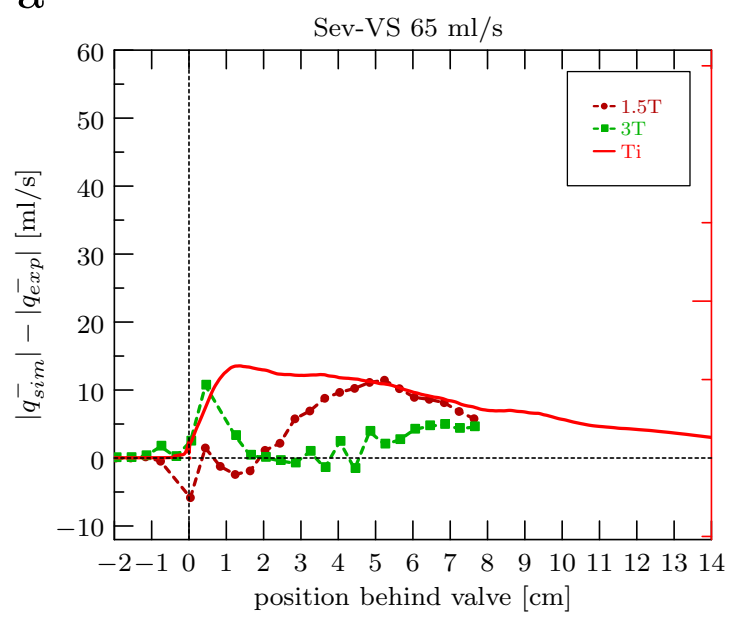

$\mathrm{C}$

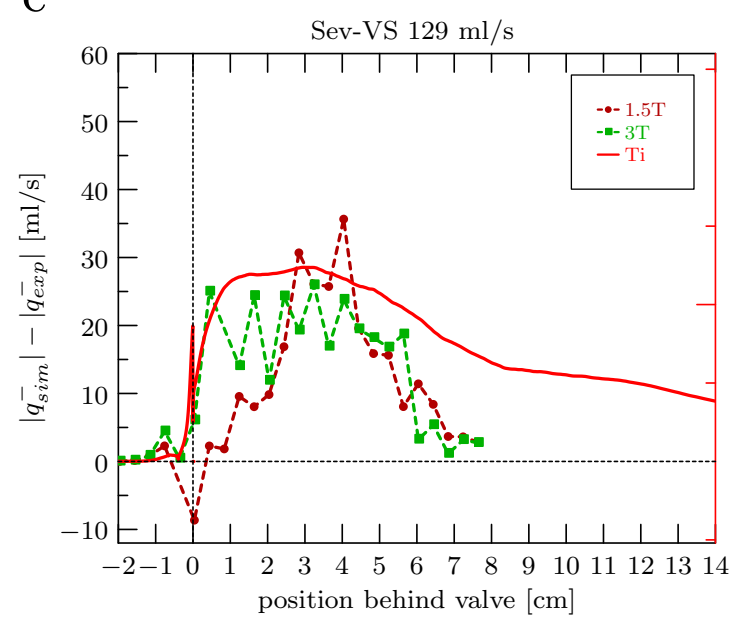

$\mathrm{b}$

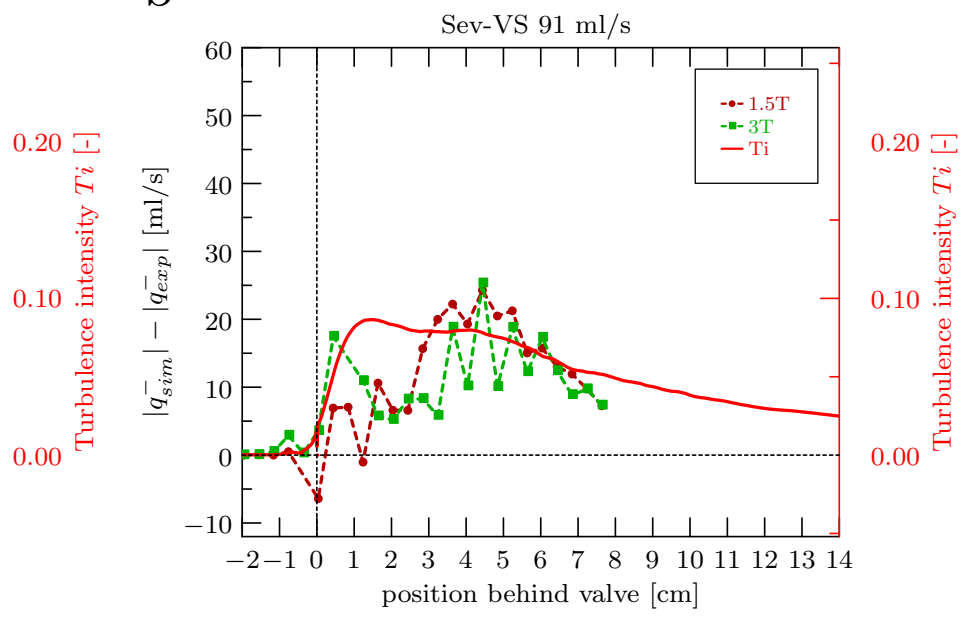

d

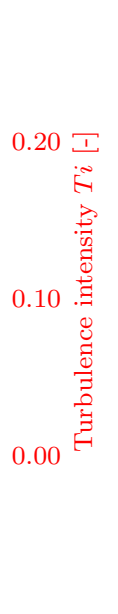

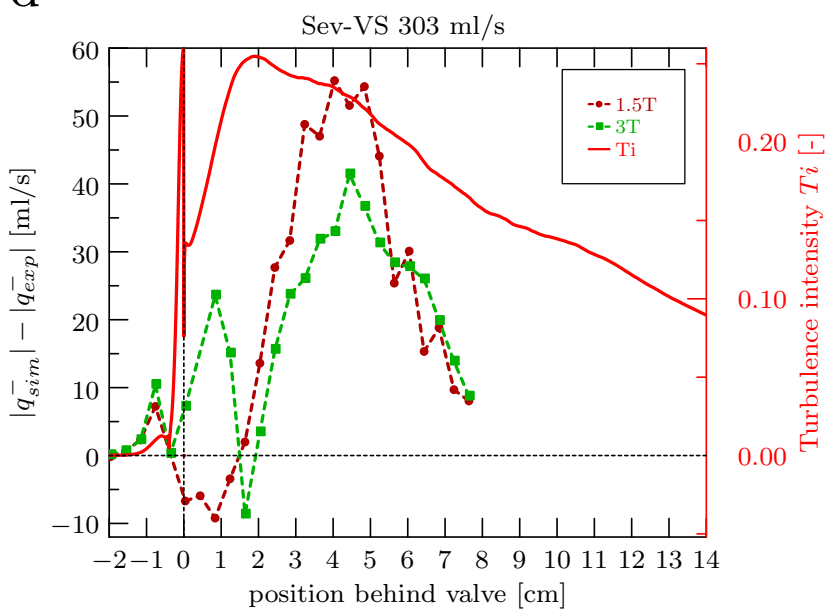

Fig. 7 Horizontal profiles of the difference between experimentally (PC-MRI) and computationally (LBM) obtained magnitudes of backward fluxes $\left|q_{\text {exp }}^{-}\right|$and $\left|q_{\text {sim }}^{-}\right|$, respectively, together with the spatially averaged turbulence intensity for all four flow rates for the severely-stenosed (Sev-VS) valve. The dotted vertical line represents the valve position. 